\title{
Resonances of a Forced Mathieu Equation with Reference to Wind Turbine Blades
}

\author{
Venkatanarayanan Ramakrishnan and Brian F Feeny \\ Dynamics Systems Laboratory: Vibration Research \\ Department of Mechanical Engineering \\ Michigan State University \\ East Lansing, Michigan 48824
}

\begin{abstract}
A horizontal axis wind turbine blade in steady rotation endures cyclic transverse loading due to wind shear, tower shadowing and gravity, and a cyclic gravitational axial loading at the same fundamental frequency. These direct and parametric excitations motivate the consideration of a forced Mathieu equation with cubic nonlinearity to model its dynamic behavior. This equation is analyzed for resonances by using the method of multiple scales. Superharmonic and subharmonic resonances occur. The effect of various parameters on the response of the system is demonstrated using the amplitude-frequency curve. Order-two superharmonic resonance persists for the linear system. While the order-two subharmonic response level is dependent on the ratio of parametric excitation and damping, nonlinearity is essential for the order-two subharmonic resonance. Order-three resonances are present in the system as well and they are similar to those of the Duffing equation.
\end{abstract}

\section{Introduction}

The blades of a horizontal axis wind turbine rotate through the field of gravity and undergo aerodynamic loads. Under steady operation and wind conditions, in which the blade rotates at a constant rate $\Omega$, a blade takes on cyclic transverse loading due to both gravity and aerodynamic forces with effects of wind shear and tower passing. The blade also endures gravitational axial compression (softening) when pointing up, and axial tensioning (stiffening) when pointing down. Considering these effects on a single-degree-of-freedom description of transverse deflection motivates us to study a forced Mathieu equation of the form

$$
\ddot{q}+\epsilon \mu \dot{q}+\left(\omega^{2}+\epsilon \gamma \cos \Omega t\right) q+\epsilon \alpha q^{3}=F \sin \Omega t,
$$


where $\omega$ plays the role of the undamped natural frequency based on the mean stiffness-tomass ratio, $\epsilon \gamma$ is the magnitude of the parametric excitation corresponding to the cyclic variation in stiffness, $F$ is the magnitude of direct cyclic forcing due to transverse gravitational and aerodynamic loading, $\Omega$ is the fundamental frequency of cyclic loadings, based on the rotation rate of the rotor, and $\epsilon \mu$ represents linear viscous damping. Parameter $\alpha$ is the coefficient of cubic nonlinearity expected due to large deformation effects, and $\epsilon$ is a small bookkeeping parameter.

There have been extensive studies on systems with parametric excitation that fit in into a minor variation of the Mathieu equation. Rhoads and Shaw [1] have studied MEMS structures with parametric amplification, in which direct excitation occurs at half the frequency of excitation as the parametric excitation. Parametric amplification was also demonstrated in experiments [2]. Other work has examined nonlinear variations of the Mathieu equation, including van der Pol, Rayleigh oscillators and the Duffing nonlinear terms. Rand et al. [3-6] analyzed the dynamics and bifucations of a forced Mathieu equation and properties of superharmonic 2:1 and 4:1 resonances. Belhaq [7] studied quasi-periodicity in systems with parametric and external excitation. Veerman [8] analyzed the dynamic response of the van der-Pol Mathieu equation. Arrowsmith [9] and Marathe [10] studied at the stability regions for the Mathieu equation. Reference [11] is an extensive compilation of the various fields of study in which the characteristics of the Mathieu equation are found and employed.

In studying equation (1), we omit effects of other terms specific to the rotating beam model, as our attention is drawn to this more fundamental equation in dynamical systems. Indeed, full partial differential equations (PDEs) of the rotating beam can be developed and modally reduced. This development is underway for transverse deflections and higher dimensional deflections as well. The transverse deflections give rise to a single assumedmode ordinary differential equation (ODE) with elements of equation (1) [12]. Numerous studies have also been done on developing differential equations of motion that represent blade motion. These traditionally include work on helicopter blades [13-16], and more recent literature is available on wind turbine blades with aerodynamic loadings and also accommodating gravity and pitching effects [17-22]. 


\section{Resonances of the Forced Nonlinear Mathieu Equa- tion}

We seek approximate solutions to equation (1) by using the method of multiple scales (MMS) $[23,24]$. The analysis reveals the existence of various sub-harmonic and super-harmonic resonances. We unfold these resonance cases and identify the critical ones for wind turbines during normal operation.

When the forcing in equation (1) is of order $\epsilon$, the analysis will indicate a primary resonance. But, this resonance is the same as in the Duffing equation, and is not treated here. The forcing in the above expression is of order one - also known as hard forcing. This will help us unfold secondary resonances. Using MMS, we incorporate fast and slow time scales $\left(T_{0}, T_{1}\right)$ and also variations in amplitude. This allows for a dominant solution $q_{0}$ and a variation of that solution $q_{1}$, i.e. such that

$$
q=q_{0}\left(T_{0}, T_{1}\right)+\epsilon q_{1}\left(T_{0}, T_{1}\right)
$$

where $T_{i}=\epsilon^{i} T_{0}$. Then $\frac{d}{d t}=D_{0}+\epsilon D_{1}$ and $D_{i}=\frac{d}{d T_{i}}$.

We substitute this formulation into equation (1) and then simplify and extract coefficients of $\epsilon^{0}, \epsilon^{1}$. The expression for co-efficient of $\epsilon^{0}$ is

$$
D_{0}^{2} q_{0}+\omega^{2} q_{0}=F \sin \Omega T_{0}
$$

The solution for this is

$$
q_{0}=A e^{i \omega T_{0}}+\Lambda e^{-i \Omega T_{0}}+\text { c.c. }
$$

where $\Lambda=\frac{F}{2\left(\Omega^{2}-\omega^{2}\right)}$, and $A=\frac{1}{2} a e^{i \beta}$.

The expression for the co-efficient of $\epsilon^{1}$ is

$$
D_{0}^{2} q_{1}+\omega^{2} q_{1}=-\mu D_{0} q_{0}-2 D_{0} D_{1} q_{0}-\gamma q_{0} \cos \Omega t-\alpha q_{0}{ }^{3}
$$

Substituting the solution for $q_{0}$ from equation (2), we expand the terms on the right hand side. We need to eliminate co-efficients of $e^{i \omega T_{0}}$ that constitute the secular terms and would make the solutions unbounded. The solvability condition is thus set by equating the co-efficients of $e^{i \omega T_{0}}$ terms to zero. 


\section{$2.1 \quad$ Non-Resonant Case}

If there is no specific relation between $\Omega$ and the natural frequency $(\omega)$ of the system, then an analysis of the solvability condition leads to the conclusion that $a \rightarrow 0$ at steady-state solution. Hence there is no effect of the nonlinear terms in the non-resonant case.

\subsection{Superharmonic Resonances}

\subsection{1 $2 \Omega \approx \omega$}

If $2 \Omega \approx \omega$, then the $\epsilon \alpha \cos \Omega t$ term and the nonlinearity from equation (3) contribute to the secular terms. We detune the frequency of excitation such that $2 \Omega=\omega+\epsilon \sigma_{2}$. In this case the solvability condition can be written as

$$
-2 A^{\prime} i \omega-\mu A i \omega-\alpha\left(3 A^{2} \bar{A}+6 \Lambda^{2} A\right)-\frac{\gamma \Lambda}{2} \exp i \sigma_{2} T_{1}=0
$$

Letting $A=\frac{1}{2} a e^{i \beta}$ and $\phi=\sigma T_{1}-\beta$, the real and imaginary parts of eqn. (7) lead to

$$
\begin{aligned}
& \text { Re }: a \phi^{\prime} \omega-\sigma \omega a+3 \alpha a\left(\Lambda^{2}+\frac{a^{2}}{8}\right)+\frac{\gamma \Lambda}{2} \cos \phi=0 \\
& I m: a^{\prime} \omega+\frac{\mu a \omega}{2}+\frac{\gamma \Lambda}{2} \sin \phi=0
\end{aligned}
$$

At steady-state the relationship between the response amplitude and the detuning parameter $\sigma_{2}$ is

$$
\sigma_{2}=3 \alpha \Lambda^{2}+\frac{3}{8} \alpha a^{2} \pm\left(\frac{\gamma^{2} \Lambda^{2}}{4 a^{2}}-\mu^{2}\right)^{1 / 2}
$$

The peak amplitude would be

$$
a_{p}=\frac{\gamma \Lambda}{2 \mu}
$$

The corresponding value of $\sigma_{2}$ is

$$
\sigma_{2 p}=3 \alpha \Lambda^{2}\left(1+\frac{\gamma^{2}}{32 \mu^{2}}\right)
$$

Hence we can conclude the following:

- the peak value is independent of $\alpha$,

- $\alpha, \Lambda$ and $\gamma / \mu$ affect the peak location and as they increase, $\left|\sigma_{2 p}\right|$ increases,

- the sign of $\alpha$ determines the sign of $\sigma_{p}$. 


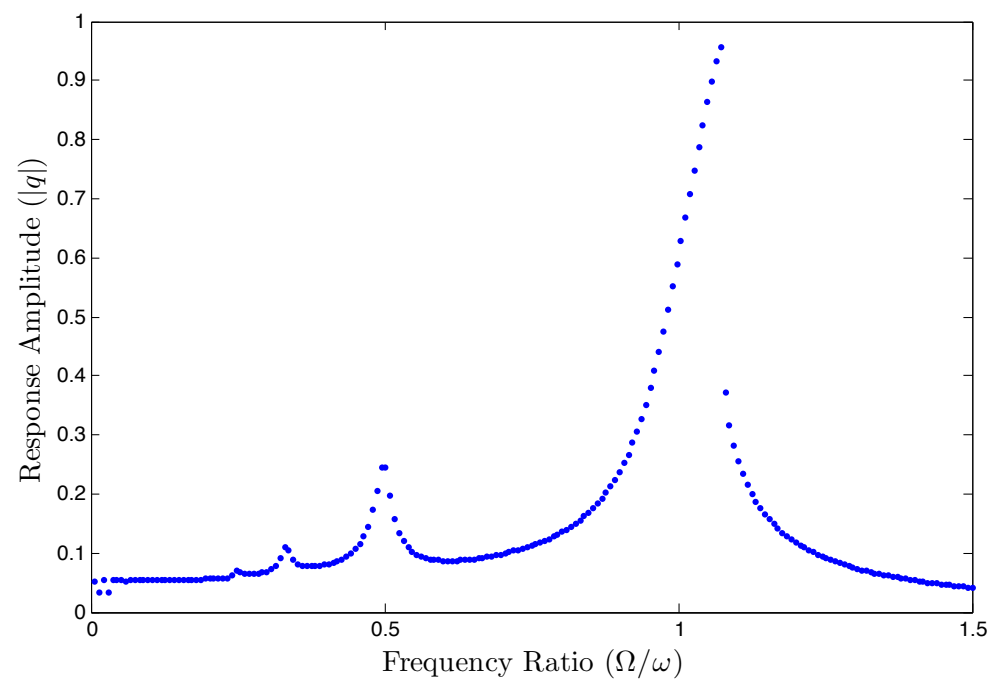

Figure 1: Simulated response of equation (1) showing superharmonic resonances at orders $1 / 2$ and $1 / 3 ; \epsilon=0.1, \mu=0.5, \Omega=0.5, \alpha=3, F=0.05, \gamma=3$. The frequency ratio sweeps up.

Vertical tangencies in the curve of equation (5) can be found and shown to coincide with stability transitions, such that the unstable branch lies between the vertical tangencies.

A sample response of equation (1) is numerically simulated. The response curve shown in Figure 1 has the primary frequency and two superharmonics shown at $1 / 3$ and $1 / 2$ the natural frequency. Superharmonic response at this order is also noticed for a linear system (see Figure 2). This agrees with our calculation of peak amplitude (see equation (6)). When nonlinearity $\alpha=0$, this superharmonic resonance peak still persists.

Wind turbine blades are generally designed such that the natural frequency of the rotating blade in lead-lag (in-plane) motion is below the rotational frequency. This analysis implies potential existence of superharmonic resonances which would also provide additional critical frequencies where the response of the blade would be dominant. Such responses would increase loading on the gearbox and other components and increased bending on the blades.

\subsection{2 $3 \Omega \approx \omega$}

If $3 \Omega \approx \omega$, the cubic nonlinearity contributes to the secular terms. We detune the frequency of excitation such that $3 \Omega=\omega+\epsilon \sigma$.

The solvability condition will have additional terms that have $3 \Omega T_{0}$ as an exponential 


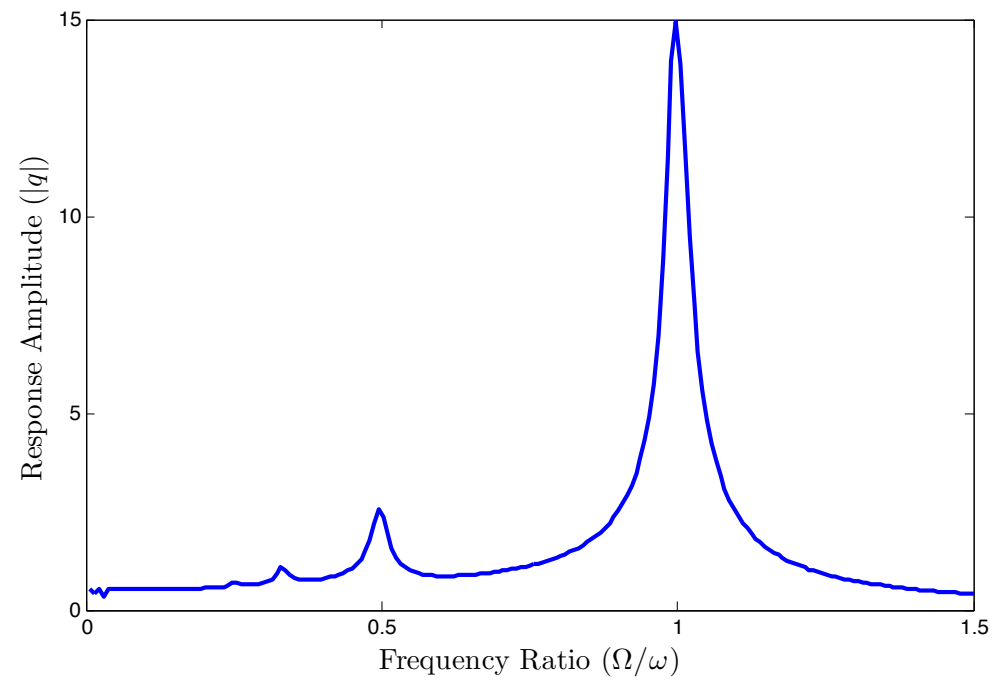

Figure 2: Simulated response of the linear case of equation (1) showing superharmonic resonances at orders $1 / 2$ and $1 / 3 ; \epsilon=0.1, \mu=0.1, \Omega=0.5, \alpha=0, F=0.5, \gamma=3$.

argument in equation (3), and it takes the form

$$
-2 A^{\prime} i \omega-\mu A i \omega-\alpha\left(3 A^{2} \bar{A}+6 \Lambda^{2} A\right)-\alpha \Lambda^{3} e^{i \sigma T_{1}}=0
$$

Following the analysis done in the previous section for the $2 \Omega$ superharmonic resonance, we substitute for $A$ and separate the equation into real and imaginary parts. Using $\phi=$ $\sigma T_{1}-\beta$, we arrive at a homogenous set of equations

$$
\begin{aligned}
& \text { Re }: a \phi^{\prime} \omega-\sigma \omega a+3 \alpha a\left(\Lambda^{2}+\frac{a^{2}}{8}\right)+\alpha \Lambda^{3} \cos \phi=0 \\
& I m: a^{\prime} \omega+\frac{\mu a \omega}{2}+\alpha \Lambda^{3} \sin \phi=0
\end{aligned}
$$

in $\phi$ and $a$.

These expressions are the same as the ones we would get in the synthesis of a standard Duffing equation with hard excitation [23]. For steady state solutions $a^{\prime}=\phi^{\prime}=0$, which is satisfied if

$$
\left[\frac{\mu}{2}^{2}+\left(\sigma-3 \alpha \Lambda^{2}-\frac{3}{8} \alpha a^{2}\right)^{2}\right] a^{2}=\frac{\alpha^{2} \Lambda^{6}}{\omega^{2}}
$$

This is a quadratic expression in the detuning parameter $\sigma$. Solving for $\sigma$ we get, 


$$
\sigma=3 \alpha \Lambda^{2}+\frac{3}{8} \alpha a^{2} \pm\left({\frac{\alpha^{2} \Lambda^{6}}{\omega^{2} a^{2}}}^{6}{\frac{\mu^{2}}{2}}^{1 / 2}\right.
$$

The peak amplitude and corresponding value of $\sigma$ are

$$
a_{p}=\frac{\alpha \Lambda^{3}}{\mu \omega}
$$

and

$$
\sigma_{p}=\frac{3 \alpha \Lambda^{2}}{\omega}\left(1+\frac{\alpha^{2} \Lambda^{4}}{8 \omega^{2} \mu^{2}}\right)
$$

Therefore, the peak amplitude and frequency of this resonance are affected by the nonlinearity via $\alpha$ and by the amplitude of excitation via $\Lambda$. The parametric excitation term does not contribute to this resonance at first order. The value of peak amplitude at this resonance is directly proportional to $\alpha$ (from equation (9)). However, numerical simulations for a linear version of our system show the persistence of this superharmonic peak (see Figure 2). Higher order perturbation analysis (not shown here) reveals the existence of this resonant condition in the absence of nonlinearity.

\subsection{Subharmonic Resonances}

\subsection{1 $\Omega \approx 2 \omega$}

If $\Omega \approx 2 \omega$ then the terms with $i(\Omega-\omega) T_{0}$ as the exponent add to the secular terms. The linear parametric excitation term and the nonlinearity contribute to this resonance. The solvability condition with these added terms is

$$
-2 A^{\prime} i \omega-\mu A i \omega-\alpha\left(3 A^{2} \bar{A}+6 \Lambda^{2} A\right)-\frac{\gamma \bar{A}}{2} \exp i \sigma T_{1}=0
$$

We substitute the exponential form for $A$, separate the real and imaginary parts, and let $\phi=\sigma T_{1}-2 \beta$, to get

$$
\begin{aligned}
& R e:-a \omega \phi^{\prime}-\sigma \omega a+3 \alpha a\left(\Lambda^{2}+\frac{a^{2}}{8}\right)+\frac{\gamma a}{4} \cos (\phi)=0 \\
& I m: a^{\prime} \omega+\frac{\mu a \omega}{2}+\frac{\gamma a}{4} \sin \left(\sigma T_{1}-2 \beta\right)=0
\end{aligned}
$$

Seeking steady state, we let $\phi^{\prime}=a^{\prime}=0$, which yields 


$$
\begin{gathered}
\frac{-\mu a}{2 \omega}=\frac{\gamma a}{4 \omega} \sin \phi \\
\frac{\sigma a}{2}-\frac{\alpha}{\omega}\left(\frac{3 a^{3}}{8}+3 \Lambda^{2} a\right)=\frac{\gamma a}{4 \omega} \cos \phi
\end{gathered}
$$

where $\phi=\sigma T_{1}-2 \beta$. We eliminate $\phi$ dependence to get the frequency response equation as

$$
\frac{\gamma^{2} a^{2}}{16 \omega^{2}}=\left(\sigma a-\frac{\alpha a}{\omega}\left(\frac{3 a^{2}}{8}+3 \Lambda^{2}\right)\right)^{2}+\frac{\mu^{2} a^{2}}{4 \omega^{2}}
$$

For this equation we have the trivial solution $a=0$ and another set of solutions of the form $a^{2}=p_{1} \pm\left(p_{1}^{2}-q_{1}\right)^{1 / 2}$, where

$$
p_{1}=\frac{3 \sigma \alpha}{4 \omega}-\frac{9 \Lambda^{2} \alpha^{2}}{2 \omega^{2}} \text { and } q_{1}=\frac{9 \alpha^{2}}{16 \omega^{2}}\left(\left(\sigma-\frac{6 \alpha \Lambda^{2}}{\omega}\right)^{2}+\frac{4 \mu^{2}-\gamma^{2}}{4 \omega^{2}}\right)
$$

These are similar in form to the standard Duffing subharmonic (presented in section 2.3.2), but the dependence on parameters differs. The nontrivial solutions for $a$ are real only when $p_{1}>0$ and $p_{1} \geq q$. These conditions imply that solutions will exist if

$$
\Lambda^{2}<\frac{\sigma \omega}{6 \alpha} \text { and } \mu^{2} \leq \frac{\gamma^{2}}{4 \omega^{2}}
$$

These are two distinct conditions on the parameters of our problem. The parametric excitation term must be sufficiently large, or the damping sufficiently small. Also, the product of the co-efficients of the nonlinear cubic term and $\Lambda^{2}$ must be sufficiently small or detuning must be sufficiently large. However, the presence of the cubic nonlinearity is essential for this subharmonic to occur.

\subsection{2 $\Omega \approx 3 \omega$}

If $\Omega \approx 3 \omega$, the cubic nonlinearity contributes to the secular terms. We detune the frequency of excitation such that $\Omega=3 \omega+\epsilon \sigma$. Then $\Omega T_{0}=(3 \omega+\epsilon \sigma) T_{0}=3 \omega T_{0}+\sigma T_{1}$.

The solvability condition takes the form

$$
-2 A^{\prime} i \omega-\mu A i \omega-\alpha\left(3 A^{2} \bar{A}+6 \Lambda^{2} A\right)-\alpha 3 \bar{A}^{2} \Lambda e^{i \sigma T_{1}}=0
$$

which is the same as that of the standard Duffing equation. We substitute the exponential form for $A$, separate the real and imaginary parts, and let $\phi=\sigma T_{1}-3 \beta$ to transform this into an autonomous system. Seeking steady state, we let $\phi^{\prime}=a^{\prime}=0$. Eliminating $\phi$ leads to the nonlinear frequency response equation 


$$
\left[\frac{9 \mu^{2}}{4}+\left(\sigma-\frac{9 \alpha \Lambda^{2}}{\omega}-\frac{9 \alpha}{8 \omega} a^{2}\right)^{2}\right] a^{2}=\frac{81 \alpha^{2} \Lambda^{2}}{16 \omega^{2}} a^{4}
$$

We recognize these as the terms that appear in the subharmonic resonance case of a Duffing equation. The solutions of equation (15) are either $a=0$ or $a^{2}=p \pm\left(p^{2}-q\right)^{1 / 2}$, where

$$
p=\frac{8 \omega \sigma}{9 \alpha}-6 \Lambda^{2} \text { and } q=\frac{64 \omega^{2}}{81 \alpha^{2}}\left[\frac{9 \mu^{2}}{4}+\left(\sigma-\frac{9 \alpha \Lambda^{2}}{\omega}\right)^{2}\right]
$$

Since $q$ is always positive, we need $p>0$ and $p^{2} \geq q$. This requires that

$$
\Lambda^{2}<\frac{4 \omega_{0} \sigma}{27 \alpha} \text { and } \frac{\alpha \Lambda^{2}}{\omega}\left(\sigma-\frac{63 \alpha \Lambda^{2}}{8 \omega}\right)-2 \mu^{2} \geq 0
$$

which can be compared to the order-two conditions (13). Then $\frac{63 \alpha \Lambda^{2}}{2 \omega \mu}=\frac{2 \sigma}{\mu} \pm\left(\frac{4 \sigma^{2}}{\mu^{2}}-63\right)^{1 / 2}$ defines the boundary of the region in the $\Lambda \sigma$ plane where non-trivial solutions can exist.

\section{Discussion}

We have shown the details of a first-order analysis of super- and subharmonic resonances for the system of interest.

At first order, the superharmonic resonance of order $1 / 3$ is the same phenomenon as in the Duffing equation. The nonlinear parameter, $\alpha$, scales the peak response, while both the nonlinear parameter and the direct excitation level affect the frequency value of the peak response. The superharmonic response of order $1 / 2$ involves interaction between the parametric excitation and both the nonlinear parameter and the direct excitation. In fact, if the nonlinearity is not present, i.e. if $\alpha=0$, this resonance persists. As such, a linear system excited both parametrically and directly at the same frequency can exhibit a superharmonic resonance. This complements linear primary resonance phenomena and linear subharmonic resonances exploited in parametric amplification $[1,2]$.

There also exists a primary resonance which is the same at first order as that of the Duffing equation. The parametric excitation, when at the same frequency as the direct excitation, does not affect primary resonance amplitude at first order.

Subharmonic resonances exist to first order at orders two and three. Order-two subharmonics involve interactions with the parametric excitation term, while order-three subhar- 


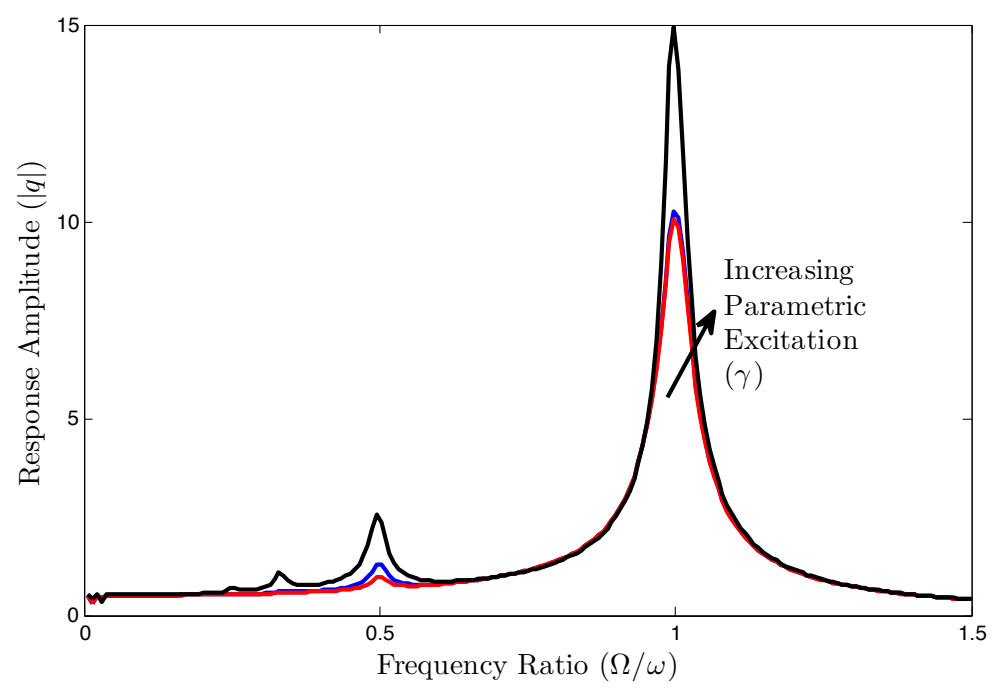

Figure 3: Simulated response of equation (1) showing the effect of the parametric forcing amplitude; $\epsilon=0.1, \mu=0.1, \Omega=0.5, \alpha=0, F=0.5$. Different curves depict $\gamma=0.5,1$ and 3.

monic resonance is the same as that of the Duffing equation to first order. The subharmonic resonance may not be critical to wind turbines, which motivate the study, as they operate below the natural frequency of the rotating blade.

The variation in system responses for changes in some parameters have been observed in simulations. Their behavior has been summarized below.

1. Effect of parametric forcing term: The system resonances increase with increased parametric forcing (Figure 3). This amplification occurs at resonances, but not between them. Beyond a certain value of $\gamma$ the system goes unstable at primary resonance. When approaching this instability, the primary resonance increases abruptly, which is not captured in the first order analysis. Ongoing work aims at unfolding this with a higher order perturbation analysis.

2. Effect of direct forcing term: As expected, an increase in the direct forcing term $F$ increases the overall magnitude of response evenly over the entire spectrum (Figure 4).

3. Effect of damping term: Increase in the damping decreases the overall magnitude of response over the entire spectrum, particularly at resonances. The superharmonic and primary resonance peaks scale as $1 / \mu$. 


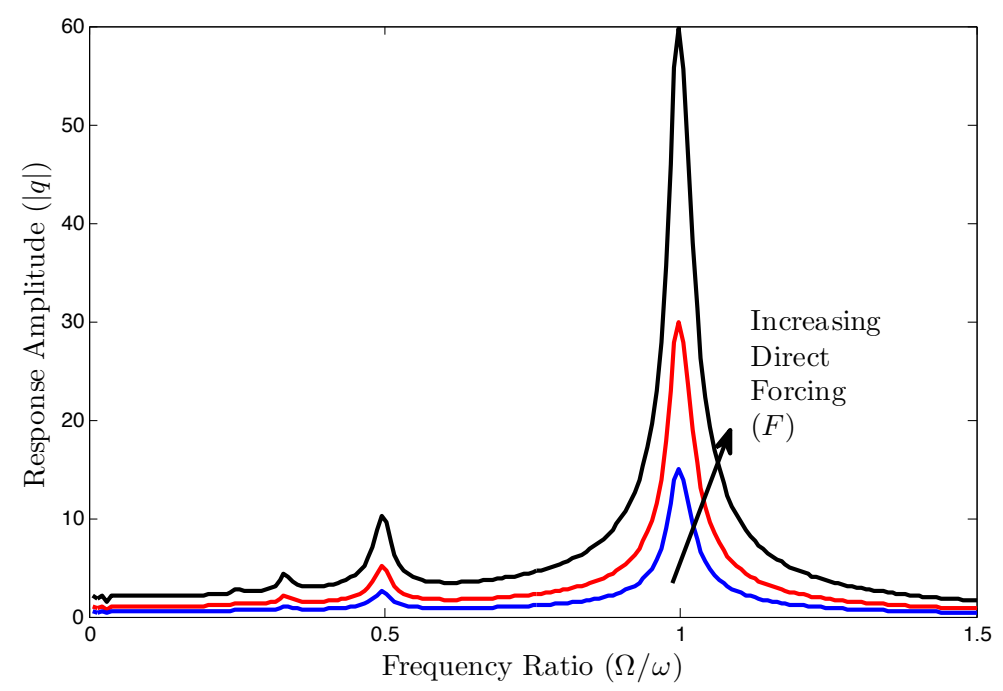

Figure 4: Simulated response of equation (1) showing the effect of the direct forcing amplitude; $\epsilon=0.1, \mu=0.1, \Omega=0.5, \alpha=0, \gamma=3$. Different curves depict $\mathrm{F}=0.5,1$ and 2 .

4. Effect of nonlinearity: An increase in nonlinearity $(\alpha)$ causes the response curve to bend over more significantly. This bending can induce jump instabilities as the frequency slowly varies. Also, in the presence of strong enough nonlinearity, the response amplitude of the superharmonic of order two is of a similar order of magnitude as the primary resonance, as seen in Figure 5.

\section{Conclusion}

We have studied the forced Mathieu equation, in which the direct and parametric excitations occur at the same frequency, out of phase by 90 degrees, and which includes a cubic nonlinearity. Perturbation analysis of equation (1) revealed the presence of multiple superand sub-harmonic resonances. The superharmonic resonance of order $1 / 2$ persists for the linear Mathieu equation with direct forcing.

This brief note has focused on resonances, and not stability. The Mathieu equation is well known to have instabilities in the space of stiffness and parametric excitation parameters. We have observed instabilities at primary resonance in simulations. A higher-order analysis is underway to describe stability transitions, as well as higher-order effects on resonances.

The presence of superharmonic resonances may be significant for wind turbines, which 


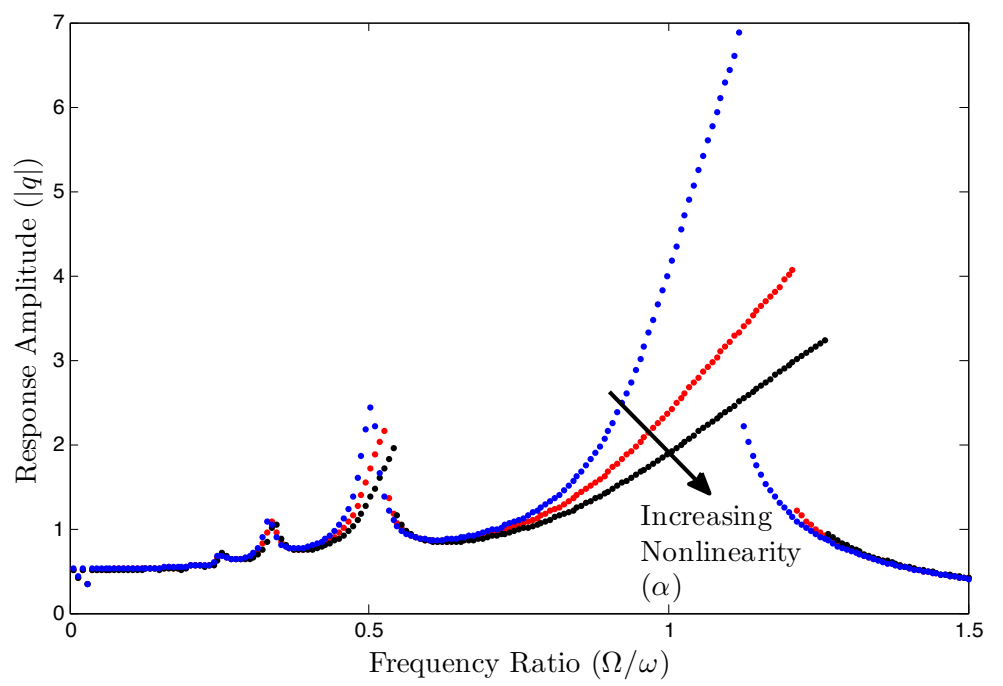

Figure 5: Simulated sweep-up response of equation (1) showing the effect of the nonlinear term; $\epsilon=0.1, \mu=0.1, \Omega=0.5, F=0.5, \gamma=3$. Different curves depict $\alpha=0.1,0.5$ and 1 .

are designed to operate below the lowest natural frequency. Further study is also underway to bring a more complete picture for the interpretation of wind turbine blade dynamics. This ongoing work includes the development of the nonlinear in-plane partial differential equation, and its assumed-mode projection, as well as an out-of-plane model, with high fidelity aerodynamic loading conditions. These models will have additional nonlinear terms, and may have restrictions on the use of the small parameter.

\section{Acknowledgment}

This material is based on work supported by National Science Foundation grant (CBET0933292). Any opinions, findings and conclusions or recommendations expressed are those of the authors and do not necessarily reflect the views of the NSF.

\section{References}

[1] Rhoads, J., and Shaw, S., 2010. "The impact of nonlinearity on degenerate parametric amplifiers". Applied Physics Letters, 96(23), June. 
[2] Rhoads, J., Miller, N., Shaw, S., and Feeny, B., 2008. "Mechanical domain parametric amplification". Journal of Vibration and Acoustics, 130(6), December, p. 061006(7 pages).

[3] Pandey, M., Rand, R., and Zehnder, A. T., 2007. "Frequency locking in a forced Mathieu-van der Pol-Duffing system". Nonlinear Dynamics, 54(1-2), February, pp. 312.

[4] Month, L., and Rand, R., 1982. "Bifurcation of 4-1 subharmonics in the non-linear Mathieu equation". Mechanics Research Communication, 9(4), pp. 233-240.

[5] Newman, W. I., Rand, R., and Newman, A. L., 1999. "Dynamics of a nonlinear parametrically excited partial differential equation". Chaos, $9(1)$, March, pp. 242-253.

[6] Ng, L., and Rand, R., 2002. "Bifurcations in a Mathieu equation with cubic nonlinearities". Chaos Solitions and Fractals, 14(2), August, pp. 173-181.

[7] Belhaq, M., and Houssni, M., 1999. "Quasi-periodic oscillations, chaos and suppression of chaos in a nonlinear oscillator driven by parametric and external excitations". Nonlinear Dynamics, 18(1), June, pp. 1-24.

[8] Veerman, F., and Verhulst, F., 2009. "Quasiperiodic phenomena in the van der PolMathieu equation". Journal of Sound and Vibration, 326(1-2), September, pp. 314320 .

[9] Arrowsmith, D., and Mondragón, R., 1999. "Stability region control for a parametrically forced Mathieu equation". Mecannica, 34, December, pp. 401-410.

[10] Marathe, A., and Chatterjee, A., 2006. "Asymmetric Mathieu equations". Proceedings of The Royal Society A(462), February, pp. 1643-1659.

[11] McLachlan, N., 1964. Theory and Application of Mathieu Functions. Dover Publications, New York.

[12] Ramakrishnan, V., and Feeny, B. F., 2011. "In-plane nonlinear dynamics of wind turbine blades". In ASME International Design Engineering Technical Conferences, 23th Biennial Conference on Vibration and Noise, pp. no. DETC2011-48219, on CDROM. 
[13] Hodges, D., and Dowell, E., 1974. Nonlinear equations of motion for elastic bending and torsion of twisted nonuniform rotor blades. Technical Note D-7818, NASA.

[14] Bhat, S. R., and Ganguli, R., 2004. "Validation of comprehensive helicopter aeroelastic analysis with experimental data". Defence Science Journal, 54(4), pp. 419-427.

[15] Wang, Y. R., and Peters, D. A., 1996. "The lifting rotor inflow mode shapes and blade flapping vibration system eigen-analysis". Computer Methods in Applied Mechanics and Engineering, 134(1-2), pp. 91-105.

[16] Rand, O., and Barkai, S. M., 1997. "A refined nonlinear analysis of pre-twisted composite blades". Composite structures, 39(1-2), pp. 39-54.

[17] Wendell, J., 1982. Simplified aeroelastic modeling of horizontal-axis wind turbines. Technical Report DOE/NASA/3303-3; NASA-CR-168109, Massachusetts Inst. of Tech., Cambridge, USA, September.

[18] Kallesøe, B. S., 2007. "Equations of motion for a rotor blade, including gravity and pitch action". Wind Energy, 10(3), February, pp. 209-230.

[19] Caruntu, D., 2008. "On nonlinear forced response of nonuniform blades". In Proceeding of the 2008 ASME Dynamic Systems and Control Conference, no. DSCC2008-2157, ASME.

[20] Chopra, I., and Dugundji, J., 1979. "Nonlinear dynamic response of a wind turbine blade". Journal of Sound and Vibration, 63(2), pp. 265-286.

[21] Jonkman, J., 2010. NWTC design codes (FAST), November.

[22] Jonkman, J., 2003. Modeling of the UAE wind turbine for refinement of FAST AD. Technical Report TP-500-34755, NREL, Golden, CO, December.

[23] Nayfeh, A. H., and Mook, D. T., 1979. Nonlinear Oscillations. Wiley Interscience Publications. John Wiley and Sons, New York.

[24] Rand, R., 2005. Lecture notes on nonlinear vibration; available at http://audiophile.tam.cornell.edu/randdocs/nlvibe52.pdf. online, Ithaca, NY. 